\title{
Pre-main-sequence stars in the young open cluster NGC $1893^{\star}$
}

\section{A spectroscopic search for candidates in the area photometrically surveyed}

\author{
A. Marco ${ }^{1}$ and I. Negueruela ${ }^{2}$ \\ 1 Dpto. de Física, Ingeniería de Sistemas y Teoría de la Señal, Universidad de Alicante, Apdo. de Correos 99, 03080, Alicante, \\ Spain \\ 2 Observatoire de Strasbourg, 11 rue de l'Université, 67000 Strasbourg, France
}

Received 6 February 2002 / Accepted 28 June 2002

\begin{abstract}
We present low-resolution spectroscopy of the majority of the stars in the field of NGC 1893 which were classified as non-members based on the photometry of Marco et al. (2001). We separate field stars from pre-main-sequence members based on a combination of spectral type and photometric indices which allows us to determine the reddening to the objects. For candidates to pre-main-sequence objects, we present intermediate-resolution spectra. We confirm 3 F-type emission-line objects as massive T Tauri stars in NGC 1893 and find two good candidates for being absorption-line Herbig A stars. A B5 star is a very good candidate for an absorption-line Herbig B star. We also investigate the nature of emission-line B-type stars close to the cluster centre. Available evidence suggests that both classical Be stars and Herbig Be stars are present in NGC 1893.
\end{abstract}

Key words. open clusters and associations: individual: NGC 1893 - stars: pre-main sequence - stars: emission line, Be stars: early-type

\section{Introduction}

The very young open cluster NGC 1893 is considered to be the centre of the Aur OB2 association. NGC 1893 can be recognized as an extended region of loosely grouped early-type stars, associated with the HiI region IC 410 and obscured by several conspicuous dust clouds. NGC 1893 contains at least five O-type stars, of which two, HD 242908 (S4R2N17 in the notation used here, after Cuffey 1973) and LS V $+33^{\circ} 16$ (S3R2N15) are main-sequence O5 stars, and therefore younger than $~ 3 \mathrm{Myr}$.

In a recent work (Marco et al. 2001, henceforth Paper I), we used $u b v y \mathrm{H} \beta \mathrm{CCD}$ photometry of the central area of NGC 1893 (four partially overlapping 5'.6 $\times$ 5'.6 frames covering a total area of approximately $8^{\prime} \times 15^{\prime}$ ) to identify $\sim 40$ very likely members, all of them close to the main sequence (MS) and with spectral types in the B0-A0 range. From the members selected, we derived an average colour excess $E(b-y)=0.33 \pm$ 0.03 and a dereddened distance modulus $V_{0}-M_{V}=13.9 \pm$ 0.2 (corresponding to the distance of $6 \mathrm{kpc}$ ) for the cluster, though some stars presented rather higher reddenings. We also

Send offprint requests to: A. Marco, e-mail: amparo@astronomia.disc.ua.es

* Based on observations obtained at the Asiago Observatory (Italy), the Isaac Newton Telescope (La Palma, Spain) and Observatoire de Haute Provence (CNRS, France). identified five emission-line stars as likely pre-main-sequence (PMS) members of NGC 1893.

The presence of PMS stars in NGC 1893 is not surprising, given its youth, and had already been suggested by Vallenari et al. (1999), based on an infrared study of the region. However, once the presence of emission-line PMS objects has been confirmed, one has to face the possibility that some of the stars rejected as field interlopers may actually be PMS stars in NGC 1893. This has no impact on the procedure followed in Paper I, where the purpose was identifying MS members for ZAMS fitting. The detection of PMS objects in NGC 1893, though, is interesting on its own, because NGC 1893 is one of the youngest open clusters known and has a moderately large population of O-type stars, representing thus a good laboratory for the study of massive star formation and the impact of massive stars on the formation of lower-mass stars.

In this paper, we pursue this aim by combining the accurate Strömgren photometry from Paper I with spectral classifications based on new spectroscopic observations. This method allows us to separate foreground stars from PMS stars in NGC 1893.

\section{Observations}

We obtained low-resolution spectra of 25 stars in the field of NGC 1893 during a run from November 29th to 
Table 1. Log of intermediate resolution spectroscopic observations. The October 2001 observations were taken with the OHP 1.93-m. All other observations are from the INT.

\begin{tabular}{|c|c|c|c|}
\hline Star & $\begin{array}{c}\text { Date of } \\
\text { observation }\end{array}$ & Resolution & $\lambda$ Range \\
\hline S1R2N4 & 2001 Feb. 8 & $1.4 \AA /$ pixel & $4200-7200 \AA$ \\
\hline S1R2N23 & 2001 Oct. 25 & $1.8 \AA ̊ ̊ /$ pixel & $3600-6900 \AA$ \\
\hline S2R1N16 & 2000 Nov. 17 & $1.4 \AA ̊ ̊ / p i x e l$ & $4200-7200 \AA$ \\
\hline S2R1N26 & 2001 Oct. 25 & $1.8 \AA ̊ ̊ / p i x e l$ & $3600-6900 \AA$ \\
\hline S3R1N3 & 2000 Nov. 17 & $1.4 \AA ̊ ̊ / p i x e l$ & $4200-7200 \AA$ \\
\hline S3R1N3 & 2001 Feb. 8 & $0.4 \AA /$ pixel & $3950-5000 \AA$ \\
\hline S3R1N4 & 2000 Nov. 17 & $1.4 \AA$ A pixel & $4200-7200 \AA$ \\
\hline S3R1N4 & 200 & $0.4 \AA /$ pixel & $3950-5000 \AA$ \\
\hline S3R1N9 & 2001 Oct. 25 & $1.8 \AA ̊ ̊ /$ pixel & $3600-6900 \AA$ \\
\hline S3R1N12 & 2001 Oct. 25 & $1.8 \AA ̊ ̊ /$ pixel & $3600-6900 \AA$ \\
\hline S3R1N13 & 2001 Oct. 25 & $1.8 \AA ̊ ̊ /$ pixel & $3600-6900 \AA$ \\
\hline S3R2N6 & 2001 Oct. 25 & $1.8 \AA ̊ ̊ /$ pixel & $3600-6900 \AA$ \\
\hline S3R2N7 & 2001 Oct. 25 & $1.8 \AA ̊ /$ pixel & $3600-6900 \AA$ \\
\hline S3R2N12 & 2001 Oct. 25 & $1.8 \AA ̊ ̊ /$ pixel & $3600-6900 \AA$ \\
\hline S4R2N28 & 2001 Oct. 25 & $1.8 \AA ̊ /$ pixel & $3600-6900 \AA$ \\
\hline S5001 & 2001 Feb. 8 & $1.4 \AA$ ̊/pixel & $4200-7200 \AA$ \\
\hline
\end{tabular}

December 1st 2000, using the 1.82-m telescope operated by the Osservatorio Astronomico di Padova atop of Mount Ekar, Asiago (Italy). The telescope was equipped with the Asiago Faint Object Spectrograph and Camera (AFOSC) and a 1024× 1024 pixel SiTE thinned CCD. We used grism \#4 which gives a nominal dispersion of $4.3 \AA /$ pixel (resolution $\approx 8.5 \AA$ ) over the $\lambda \lambda$ 3500-7500 A range.

Intermediate-resolution spectroscopy of selected sources was obtained using the 2.5-m Isaac Newton Telescope (INT) at La Palma, Spain. Observations were obtained in service mode on 7th November 2000 and during a two-night run in February 7-8th 2001. The telescope was equipped with the Intermediate Dispersion Spectrograph (IDS) and the 235-mm camera. The detector used was a $2048 \times 4096$ EEV10 CCD. The slit width was set to $1^{\prime \prime} .5$ and the R400V and R1200B gratings were used. The R400V grating gives a nominal dispersion of $1.4 \AA /$ pixel with the set-up selected. The wavelength range covered was $\lambda \lambda$ 4200-7200 $\AA$ during the February 2001 run and $\lambda \lambda$ 4000-7000 $\AA$ during the November 2000 observations. The R1200B grating gives a nominal dispersion of $0.4 \AA /$ pixel with our set-up, covering the wavelength range

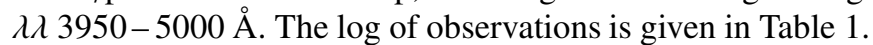

A few more spectra were obtained on October 25th 2001, using the $1.93-\mathrm{m}$ telescope at the Observatoire de Haute Provence (OHP), France. The telescope was equipped with the long-slit spectrograph Carelec and a $1024 \times 2048$ pixel EEV CCD. The $300 \mathrm{ln} \mathrm{mm} \mathrm{mm}^{-1}$ grating was used, giving nominal dispersion of $\approx 1.8 \AA$ /pixel over the $\lambda \lambda 3600-6900 \AA$ range. These observations are also listed in Table 1.

All the observations have been reduced, following standard procedures for the bias subtraction, flat-fielding and extraction, using the Starlink software packages CCDPACK

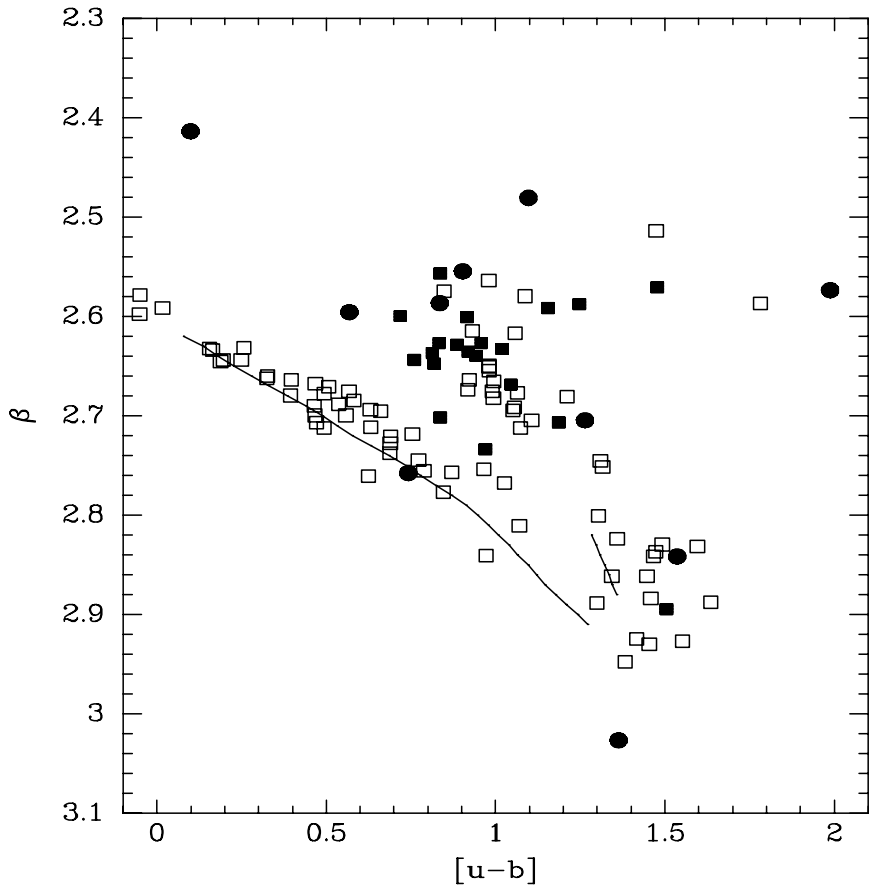

Fig. 1. The $\beta-[u-b]$ diagram for the stars in the field of NGC 1893 observed in Paper I. Filled squares are stars investigated here which turn out to be non-members. Filled circles are stars considered as PMS candidates.

(Draper et al. 2000) and FIGARo (Shortridge et al. 1997) and analysed using FIGARo and DIPSo (Howarth et al. 1998). Sky was always subtracted by fitting a low-degree polynomial to points in two regions on each side of the spectra, as implemented by the FIGARO routine POLYSKY. The size of these regions and their distance to the spectrum were selected in order to reduce the contamination due to nebular emission. Bright sky lines coming from diffuse nebular emission are visible in almost all of our spectra.

\section{Candidate selection}

PMS stars lie away from the MS in photometric diagrams and cannot be easily distinguished from field interlopers. On the other hand, the combination of accurate photometric data with spectral types derived from spectroscopy is a powerful tool for determining the properties of stars and decide on their membership. The criterion for membership used in this work, namely that the colours of candidates should indicate that they are as reddened as MS cluster members, is discussed below.

We have observed preferentially objects lying close to the spectroscopically confirmed emission-line PMS stars in the $[u-b]-\beta$ diagram (Paper I), though a few other stars were also observed, as they fell inside the slit by chance. Spectral types of stars in the field of NGC 1893 have been derived from their observed spectra. The method used has been direct comparison of the target spectra with spectra of several MK standard stars observed during the February 2001 run at the INT. Since the resolution of these standard spectra was much higher than that of the target spectra observed with AFOSC, they were binned to a similar resolution before carrying out the 


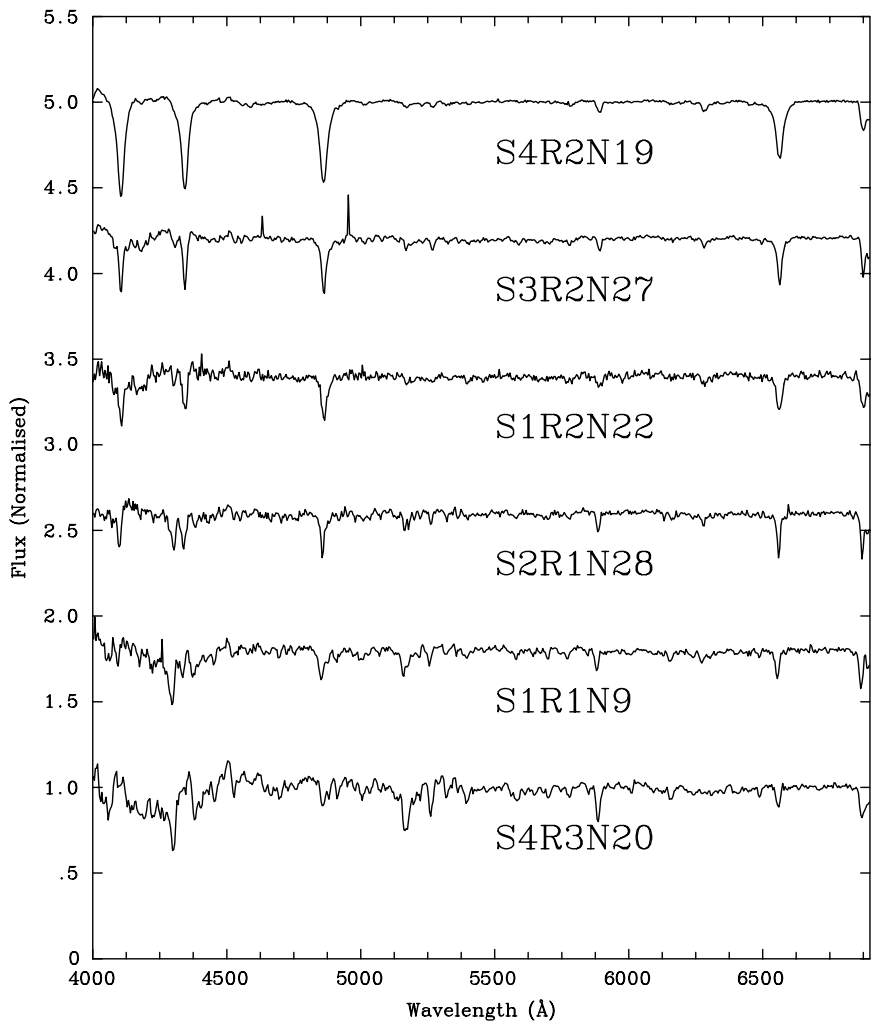

Fig. 2. Some representative spectra of field stars observed at low resolution with AFOSC at the 1.82-m telescope of Asiago observatory. For the spectral types derived, see Table 2.

Table 2. Astrophysical parameters of stars in the field of NGC 1893 which have been considered foreground stars from their spectral types and reddenings.

\begin{tabular}{lcccc}
\hline \hline Star & $V$ & $(b-y)$ & Spectral Type & $E(b-y)$ \\
\hline S1R1N9 & 14.946 & 0.540 & G3V & $\approx 0.14$ \\
S1R1N18 & 14.469 & 0.366 & F4V & $\approx 0.09$ \\
S1R2N22 & 14.183 & 0.361 & F2V & $\approx 0.12$ \\
S2R1N27 & 15.059 & 0.589 & G5V & $\approx 0.17$ \\
S2R1N28 & 15.083 & 0.477 & F8V & $\approx 0.13$ \\
S2R2N46 & 12.514 & 0.525 & G3III-V & $\lessgtr 0.12$ \\
S3R1N12 & 12.472 & 0.373 & F0III-IV & $\lessgtr 0.17$ \\
S3R2N27 & 14.204 & 0.371 & F0V & $\approx 0.15$ \\
S3R3N12 & 14.383 & 0.551 & G0V & $\approx 0.17$ \\
S4R1N1 & 14.712 & 0.519 & G5V & $\approx 0.10$ \\
S4R2N12 & 15.333 & 0.504 & F7V & $\approx 0.18$ \\
S4R2N19 & 12.284 & 0.161 & A0V & $\approx 0.14$ \\
S4R2N20 & 14.606 & 0.448 & F7V & $\approx 0.12$ \\
S4R2N22 & 13.856 & 0.342 & F6V & $\approx 0.04$ \\
S4R2N23 & 15.613 & 0.488 & G0V & $\approx 0.11$ \\
S4R2N24 & 15.820 & 0.424 & G0V & $\approx 0.04$ \\
S4R2N27 & 13.702 & 0.401 & F6V & $\approx 0.10$ \\
S4R2N29 & 14.490 & 0.591 & K1V & $\approx 0.09$ \\
S4R3N20 & 13.174 & 0.555 & K0V & $\approx 0.07$ \\
S5001 & 15.116 & 0.565 & G1V & $\approx 0.17$ \\
\hline
\end{tabular}

comparison. We followed the classification guidelines of Morgan et al. (1943), complemented by the on-line spectral atlas of Gray at the Level $5^{1}$ database.

Given the low resolution and moderate ( $\$ 50$ in most cases) Signal-to-Noise Ratio (SNR), spectral classifications derived from AFOSC spectra are probably not much more accurate than a \pm 2 subtype error. This is, however, sufficient for our purpose, i.e., the separation of field stars from PMS objects in NGC 1893. The procedure used has been as follows.

From the spectral type derived, we have computed the colour excess $E(b-y)$ for each star using the intrinsic colours $(b-y)_{0}$ corresponding to the spectral type (taken from Popper 1980 and interpolating when necessary) and the values of $(b-y)$ reported in Paper I.

It must be taken into account that none of the objects under consideration can be a MS member of NGC 1893 based on their position on the photometric diagrams (since they were selected on this basis). Therefore, if they are cluster members, they must be PMS objects with intrinsic luminosities rather higher than those corresponding to MS objects of the same spectral type. The implicit assumption is then that PMS members will have reddenings at least close to the average reddening of the cluster. This is justified, since all the MS members observed in Paper I have reddenings $E(b-y)>0.27$ when the average is $E(b-y)=0.33 \pm 0.03$. Any PMS star is likely to be surrounded by dust or some remnant of the accretion cloud and therefore will have higher reddening than an MS member. Therefore we can be confident that the individual reddening of any PMS star will be higher than the lower reddening measured for an MS member. Moreover, in principle, we have used the intrinsic colours of MS stars in the determination of $E(b-y)$, while PMS stars have higher gravities and therefore likely redder colours.

We have selected as foreground stars those objects with $E(b-y)<0.2$. This is a conservative limit in order to account for cumulative errors in the photometry, spectral types and intrinsic colour tabulation. Fortunately, there are very few doubtful cases, i.e., stars where the uncertainty in $E(b-y)$ allows the $0.2-0.3$ range. This is consistent with the idea that part of the reddening to NGC 1893 originates in its immediate vicinity. As an extra precaution, for stars with $E(b-y)$ approaching 0.2 , we checked the strength of interstellar lines (specially the large complex around $\lambda 5780 \AA$ ) and compared it with the interstellar lines on some of the MS accepted members. In all cases, we have found the lines to be much weaker in the objects whose colours correspond to foreground objects.

\subsection{Stars with Strömgren photometry}

Among the objects observed during the Asiago run, only one turned out to be clearly an emission-line star. This was S3R1N4, which had already been selected in Paper I as a possible B-type emission line object, because of its $\beta$ index. One A-type star, namely S1R2N4, displayed $\mathrm{H} \alpha$ in absorption, but extremely weak. These two objects were selected for further spectroscopy and will be discussed in Sect. 4. One bright

\footnotetext{
${ }^{1}$ nedwww.ipac.caltech.edu/level5/Gray/frames.html
} 
K-type star, S4R2N28, seemed to have extremely red colours for its spectral type, and was also selected.

Most of the objects observed, turned out to be, as expected given the large distance to the cluster, field stars. Their parameters, derived following the procedure described in the previous paragraphs, have been listed in Table 2. As can be seen from the table the reddenings to all these objects are much lower than the lowest value measured for a cluster member. The procedure is therefore very successful at separating field stars from cluster members with anomalous colours.

\subsection{Stars without Strömgren photometry}

During all observing runs, a number of objects not included in the area covered by the photometry of Paper I have been serendipetously observed, as they have fallen on the slit when we have observed other stars. Fortunately, most stars in the field have been observed by previous authors and several sets of $U B V$ photometry exist for most of them. In order to decide on the membership of these objects, we have used the CCD $U B V$ photometry of Massey et al. (1995).

Among these, two objects seemed to show weak and asymmetric $\mathrm{H} \alpha$ absorption and were selected for further study, the F-type star S3R1N9 and the A-type star S3R2N12. Both will be discussed in Sect. 4. Two other stars were only partially inside the slit and their spectra only allow approximate classification: S3R2N11 is early $K$ and therefore the measured $(B-V)=1.07$ implies $E(B-V) \lesssim 0.25$, while S2R1N29 is early $G$ and the measured $(B-V)=0.87$ implies $E(B-V) \lessgtr 0.28$. Both values are much lower than the average $E(B-V)=0.45$ for cluster members, indicating that both objects are foreground stars.

\section{Candidates observed at higher resolution}

\subsection{PMS candidates}

\subsubsection{S1R2N4}

This star was observed because the Asiago spectrum seemed to show emission infilling in $\mathrm{H} \alpha$. With the much better sky conditions of La Palma, it is easy to see that the emission actually comes from the surrounding sky area: the object is immersed in bright luminosity associated with the pennant nebula Sim 130 (Gaze \& Shajn 1952). The spectrum of this nebulosity is typical of an $\mathrm{H}$ II region and the intensity of $\mathrm{H} \alpha$ emission seems to have a local maximum at the position of S1R2N4, suggesting a physical association. We have carefully removed the sky with a polynomial fit, but it is not impossible that the Balmer lines are overcorrected (i.e., appear depeer than they really are). If the star has any intrinsic weak emission or shell spectrum, this is masked by the sky line subtraction.

The stellar spectrum is displayed in Fig. 3. The spectrum is obviously earlier than A5 and displays strong interstellar bands. Comparison with the A2V standard 70 Leo shows a similar intensity for the Balmer lines and slightly stronger metallic lines. The object is likely A3V, though A2IV is also possible. Therefore its intrinsic colour is $(b-y)_{0}=+0.04$ while the measured color is $(b-y)=+0.421$, making $E(b-y)=0.38$.

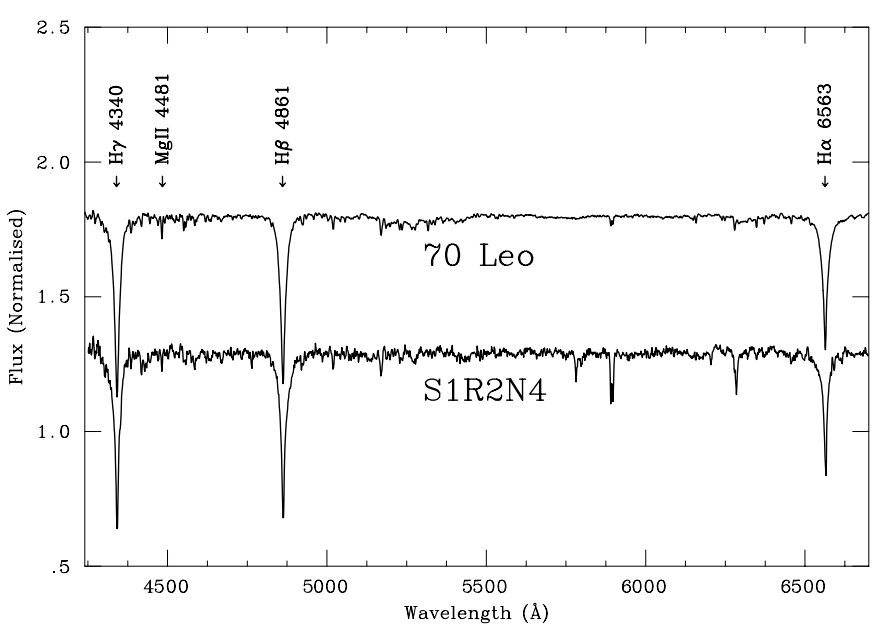

Fig. 3. Intermediate-resolution spectrum of S1R2N4, compared to the A2V standard 70 Leo. Notice the strong diffuse interstellar bands in the spectrum of S1R2N4.

The reddening is sufficiently high for it to be a member of NGC 1893. Moreover, it fulfills all the conditions to be considered a Herbig Ae star, except for the presence of emission lines, as it is associated with bright nebulosity. We favour the idea that this is a PMS star in NGC 1893. Its intrinsic luminosity is then $M_{V}=-1.7$, if the reddening is standard.

\subsubsection{S1R2N23}

S1R2N23 was selected as an emission-line object in Paper I. Its spectrum is displayed in Fig. 4. $\mathrm{H} \alpha$ is strongly in emission with an Equivalent Width $(E W)=-19.5 \pm 0.5 \AA$. $\mathrm{H} \beta$ is filledin by an emission component, while it was weakly in emission in February 2000 (Paper I). H $\delta$ appears as a broad absorption feature. The spectral classification of this object is difficult, as it seems to be covered by weak emission lines. The $\mathrm{Na} \mathrm{D}$ lines are not visible at all, but the interstellar diffuse bands are moderately strong. The strength of some metallic lines suggests a luminosity class III. The strength of the $G$ band would then support a spectral type around F6, but we feel unable to give an accurate spectral type for this object, since it does not seem to match any standard. The emission characteristics, however, indicate that it is a PMS star.

\subsubsection{S2R1N16}

This object had been selected as a PMS candidate because of the presence of emission lines in its spectrum (Paper I). Its spectral classification is in principle difficult, since - as can be seen in Fig. 4 - the Balmer lines are in emission or filled in (the $E W$ of $\mathrm{H} \alpha$ is $-7.3 \pm 0.5$ ). Based only on the metallic spectrum, a spectral type around F6 or F7 is derived. The luminosity indicators such as the Fe I $\lambda \lambda 4172,4272 \AA$ and $\mathrm{Ca}$ I $\lambda \lambda 4226 \AA$ lines suggest low luminosity (IV-V). The measured $(b-y)=+0.79$ implies then a high reddening $E(b-y) \approx 0.5$. The object is therefore an obscured PMS star in NGC 1893. 


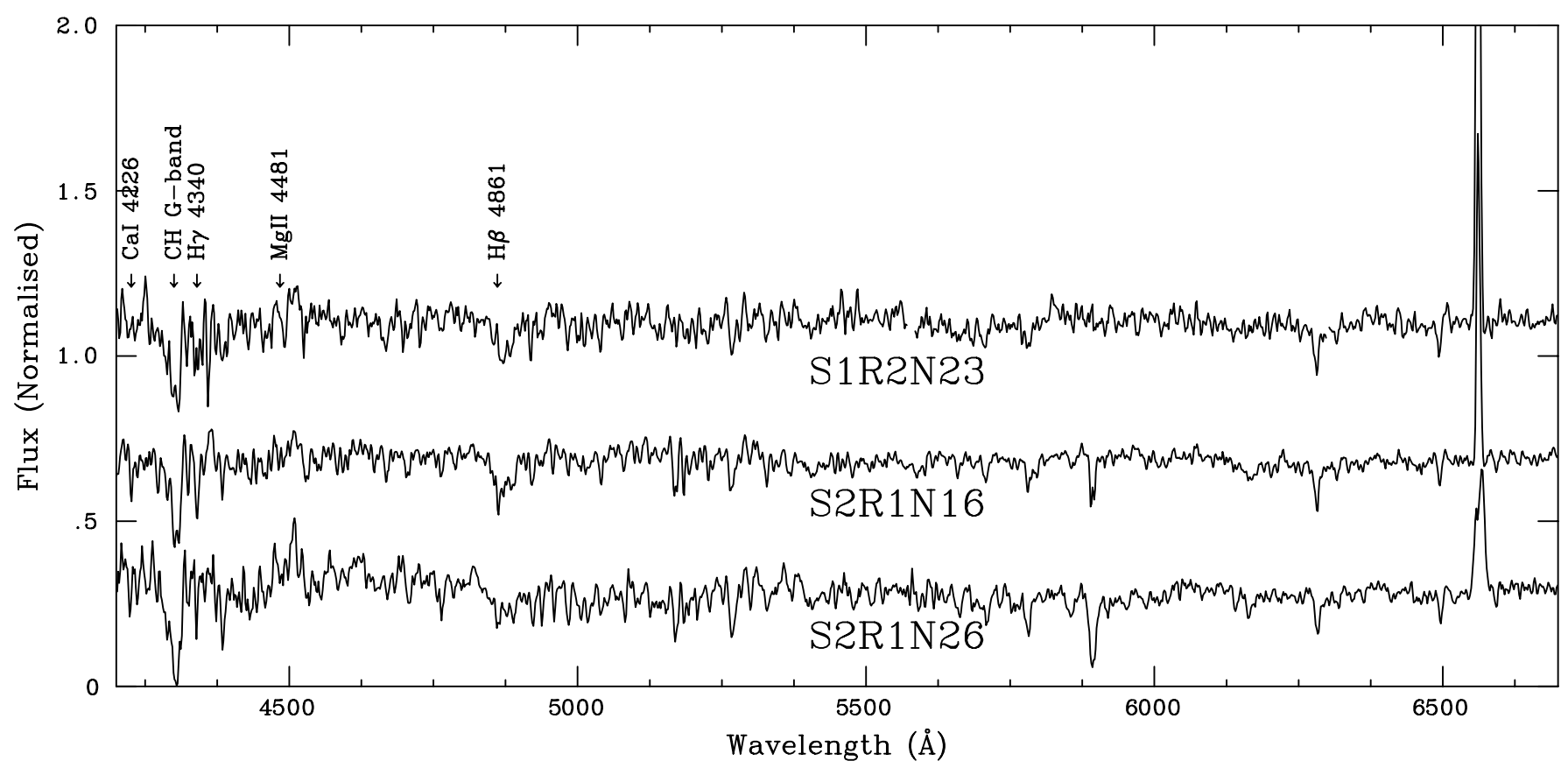

Fig. 4. Intermediate-resolution spectra of S1R2N23, S2R1N16 and S2R1N26, the three emission-line F-type stars found in Paper I. The spectra have been normalised by division into the continuum and offset by an arbitrary amount.

\subsubsection{S2R1N26}

S2R1N26 was also among the emission-line objects selected in Paper I. Its spectrum, displayed in Fig. 4, shows $\mathrm{H} \alpha$ weakly in emission $(E W=-6.0 \pm 0.5)$ and possibly double-peaked (though the subtraction of a strong sky line makes this somewhat doubtful). $\mathrm{H} \beta$ and $\mathrm{H} \gamma$ are filled-in by emission components, complicating the spectral classification. From the overall metallic spectrum, the spectral type is F9V or G0V. The measure $(b-y)=0.82$ implies then $E(b-y) \approx 0.46$, making this object an obscured PMS star in NGC 1893.

\subsubsection{S3R $1 \mathrm{~N} 9$}

S3R1N9 was selected from its Asiago spectrum, which seemed to show an emission component in $\mathrm{H} \alpha$. A higher resolution spectrum was taken with the OHP 1.93-m. No emission lines are present and the spectral type is approximately F3V. Different authors give $(B-V)$ values between 0.58 and 0.70 for S3R1N9. Even in the best case, $E(B-V) \lesssim 0.33$, meaning that S3R1N9 is a field star.

Careful inspection of the DSS2 plates of the area, however, shows that S3R1N9 has a close, faint companion, which, following the convention of Paper I, we will call S5003. The presence of a close companion may explain the variability in the values of $(B-V)$ reported in the literature, since it could probably not be separated from S3R1N9 in photoelectric photometry.

The faint companion S5003 felt partially within our slit when the OHP 1.93-m spectrum was taken. Even though its spectrum is too noisy to provide any information on its spectral type, $\mathrm{H} \alpha$ is clearly in emission. This object was likely blended with S3R1N9 in the Asiago spectrum, explaining the detection of $\mathrm{H} \alpha$ in emission. This object is likely to be a PMS star in NGC 1893 and further spectroscopy is needed.

\subsubsection{S3R1N13}

This star was identified in Paper I as having the colours of a mid-B star, but not falling on the locus of MS members of NGC 1893. Its spectrum is shown in Fig. 5. From the relative strengths of the Mg II $\lambda 4481 \AA$ and He I $\lambda 4471 \AA$, a spectral type earlier than B7 is deduced. The strength of Si II $\lambda 4128 \AA$ would suggest a B6 spectral type, if the object is on the main sequence, but from the strength of other metallic lines, a higher luminosity is inferred. We therefore assign a spectral type B5III-IV.

The measured $(b-y)=0.43$ implies then $E(b-y) \approx 0.5$ putting the object clearly at the distance of NGC 1893. Since S3R1N13 lies projected on top of IC 410, we suggest that it is a star just emerging from the parental cloud. For this reason, the reddening to this object may be anomalous, which would explain why it does not fall together with other NGC 1893 members. Assuming, for the sake of discussion, that the reddening is standard, we obtain an $M_{V}=-2.9$, clearly much higher than that of a MS star. Therefore this object is a good candidate for an absorption-line PMS B-type star in NGC 1893. Its $(V-K)=1.4$ (Tapia et al. 1991) confirms the high reddening to the star.

\subsubsection{S3R2N6}

This star forms a close apparent double with S3R2N7. Spectra of both stars are displayed in Fig. 6. The spectral type of S3R2N6 is F2V, to which a $(b-y)_{0}=+0.24$ corresponds. The measured $(b-y)=0.60$, gives $E(b-y)=0.36$, above the 


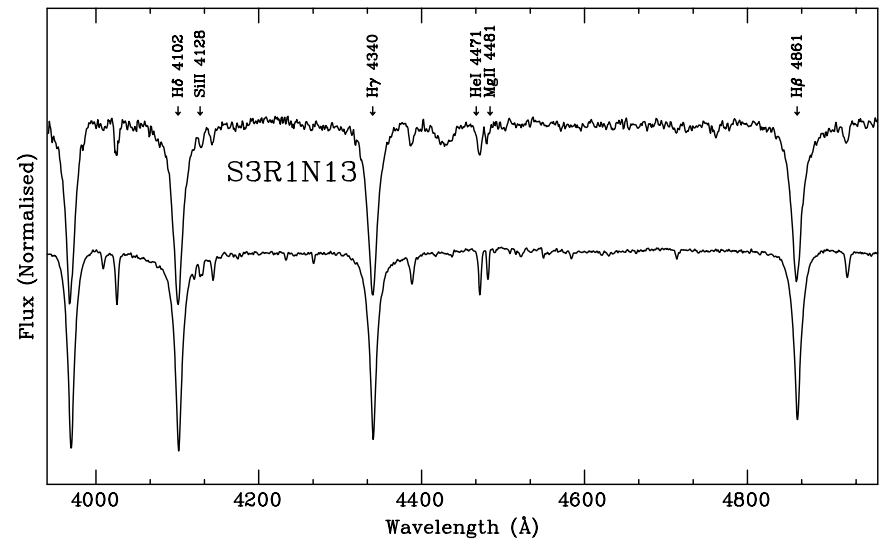

Fig. 5. Blue spectrum of S3R1N13 (top). Note the prominent interstellar diffuse bands at $\lambda \lambda 4430,4501,4726 \& 4767 \AA$. The comparison spectrum is the B6V standard 19 Tau.

average for cluster members. Photometry from Massey et al. (1995) implies $E(B-V)=0.51$, also slightly above the cluster average. If the object is a PMS star in NGC 1893, then $M_{V}=-0.7$.

S3R2N7 has a spectral type A9V. The measured $(b-y)=$ +0.43 implies $E(b-y)=0.24$, which is within our area of uncertainty. Since the intrinsic colours of stars change very quickly in this spectral range, S3R2N7 should be considered a doubtful case.

The fact that these two very close stars have similar spectral types and colours gives some weight to the possibility that both stars are PMS objects in NGC 1893.

\subsubsection{S3R2N12}

The Asiago spectrum of S3R2N12 suggested an emission component in $\mathrm{H} \alpha$. Higher-resolution spectra show that this object, like all in its vicinity, is immersed in bright diffuse nebulosity and therefore this emission could come from the sky. Inspection of images of the area shows that most of the third quadrant and specifically the area close to the O-type star HD 242926 (S3R3N1) is covered in bright diffuse nebulosity, from which these emission lines must arise. This makes extremely difficult the extraction of stellar spectra, specially when they are faint.

The spectrum of S3R2N12, shown in Fig. 6, displays unusually weak $\mathrm{H} \alpha$ absorption even when a careful sky subtraction has been made, but this is still likely to be due to sky emission. The spectral type of S3R2N12 is intermediate between the $\mathrm{A} 7 \mathrm{~V}$ and the F0V standards, and therefore an A9V spectral type seems adequate. At this spectral type $(B-V)_{0}=+0.25$ (Schmidt-Kaler 1982). Different authors provide very differing values for the magnitudes of this object, suggesting real variability. The measured $(B-V)$ varies between +0.82 (Moffat \& Vogt 1974) and +0.99 (Massey et al. 1995). Using the CCD photometry of Massey et al. (1995), we find $E(B-V)=0.74$, well above the average for cluster members. If it is a cluster member and the extinction is standard, $M_{V}=-0.9$. Therefore S3R2N12 is a good candidate to a PMS A-type star in NGC 1893.

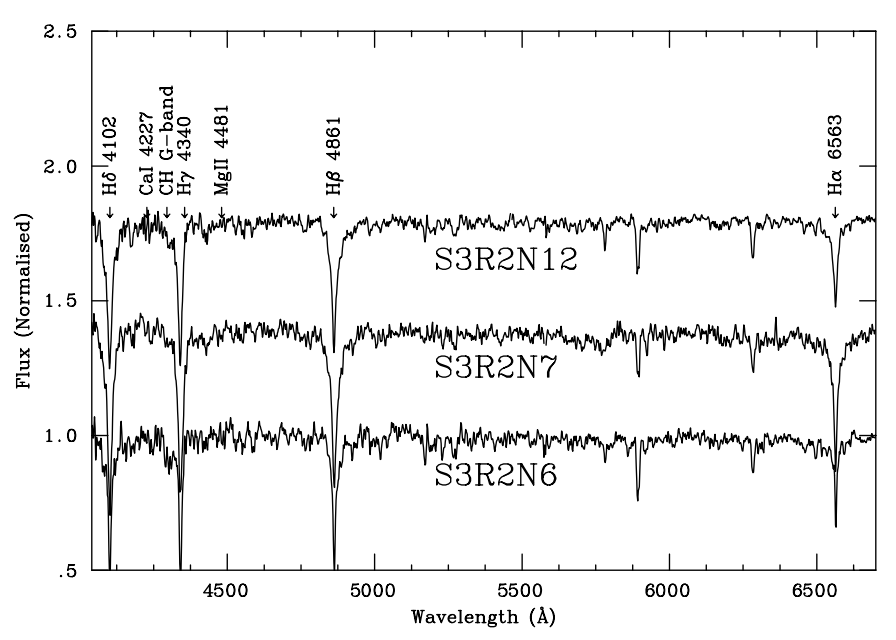

Fig. 6. Intermediate-resolution spectra of S3R2N6, S3R2N7 and S3R2N12, all of which have colours suggesting membership in NGC 1893. H $\alpha$ is unusually weak in S3R2N12, while the Balmer lines in S3R2N7 are overcorrected for sky lines.

\subsubsection{S4R2N28}

The spectrum of S4R2N28 is displayed in Fig. 7. The Balmer lines are very weak and $\mathrm{H} \delta$ is not visible at all, making this object a K-type star. No molecular bands are visible, which indicates a spectral type earlier than K5. It does not seem to be later than $\mathrm{K} 2$ and the general aspect suggests K0V. For such a spectral type, $(b-y)_{0}=+0.53$ and the measured $(b-y)=1.02$ gives an $E(b-y)=0.49$. The object must then be a cluster member. In that case, assuming standard reddening, its absolute magnitude should be $M_{V}=-2.7$.

Such a high luminosity in an object that does not show any indication of pecularities in its spectrum is surprising. On the other hand, even assuming a K2III spectral type (though all luminosity indicators argue against a luminosity higher than IV), we have $(b-y)_{0}=+0.71$ and $E(b-y)=0.31$, which still argues for membership.

Photographic $U B V$ photometry by Cuffey (1973) and Hoag et al. (1961) confirms that our photometry is not in error. Both works agree on $(B-V)=+1.7$ and $(U-B)=+1.5$, while Moffat \& Vogt (1974) give $(B-V)=+1.7$ and $(U-B)=+2.1$. In all cases, $E(B-V)=0.9$ for a K0V spectral type $\left((B-V)_{0}=\right.$ +0.8 , after Schmidt-Kaler 1982). Therefore we are certainly dealing with a star with very anomalous colours, and the possibility that it is an absorption line PMS star in NGC 1893 remains open.

\subsubsection{S5001}

The photometric results presented in Paper I indicated that this star had an $\beta$ index $<2.55$, suggestive of emission. It could not be observed from Asiago due to its nearness to a much brighter star, and therefore it was observed from the INT. The result is an spectrum without emission lines. The $\mathrm{CH}$ G band is present and its intensity is larger than that of $\mathrm{H} \gamma$. The ratio between Fe II $\lambda 4325 \AA$ and $\mathrm{H} \gamma$ gives an spectral type $\mathrm{G} 1$ or G2. Its intrinsic colour is then $(b-y)_{0}=0.4$ and the measured $(b-y)=0.57$ indicates $E(b-y)=0.17$, making it a field star. 


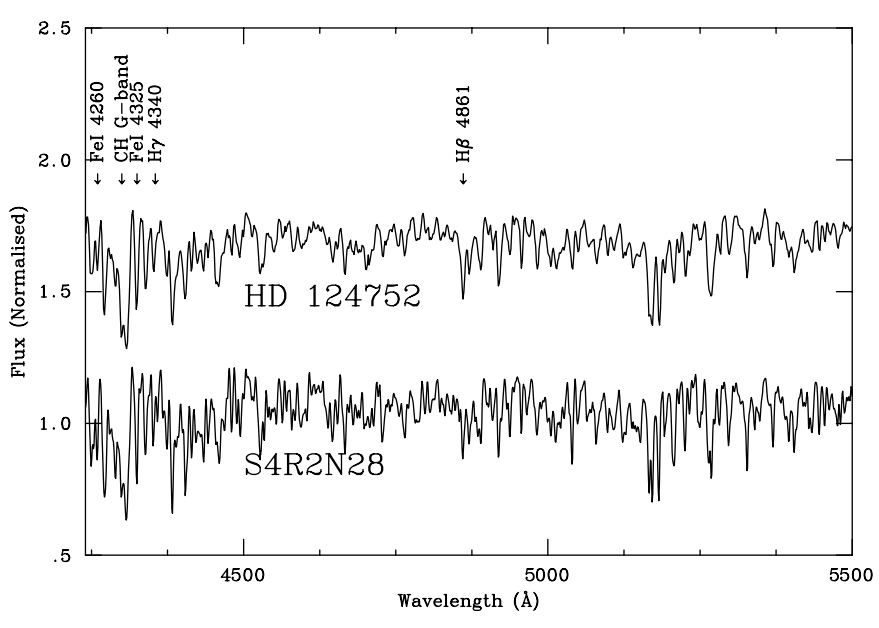

Fig. 7. Intermediate-resolution spectra of S4R2N28, compared to the K0V standard HD 124752. Metallic lines are slightly stronger in the spectrum of S4R2N28, but do not support a much higher luminosity.

\subsection{Be stars}

\subsubsection{S3R1N3}

In Paper I, this object was proposed as a Herbig Be star. Its photometric parameters suggest that it is very early. A lowresolution spectrum of this source is shown in Fig. 8, displaying very strong Balmer emission lines and a very reddened continuum.

A higher resolution spectrum is shown in Fig. 9. From the Full Width at Half Maximum (FWHM) of the three stronger He $\mathrm{r}$ lines, we estimate a rotational velocity $v \sin i \approx 340 \mathrm{~km} \mathrm{~s}^{-1}$, using the approximation discussed by Steele et al. (1999). This is only a rough estimate, because other He I lines are obviosuly filled in by emission components and the FWHM can be reflecting this effect, but the star is clearly a very fast rotator.

An exact spectral classification is difficult, because many $\mathrm{Fe}$ II emission lines, both weak and strong, blanket the photospheric spectrum. In principle, the strength of He I $\lambda 4009 \AA$ and He I $\lambda 4121 \AA$ implies a spectral type in the B0-B1 range. Given the high SNR of the spectrum, we could in principle assume that all the weak features detected are real, but the Fe II blanketing introduces spurious features. If the identifications are correct, the presence of the O II $\lambda 4242 \AA$ line indicates that the spectrum is later than B0, while the presence of $\operatorname{Si}$ Iv $\lambda 4212 \AA$ indicates that it is B0.5 or earlier. The stronger Si IV $\lambda 4089 \AA$ is not seen on the blue wing of $\mathrm{H} \delta$, but this could be due to the very large rotational broadening of the lines. The $\mathrm{O}$ II spectrum is relatively strong, suggesting that the star is B1 and/or has a luminosity class IV. We adopt a B0.5IVe spectral classification, taking into consideration that the spectral type may be off by about half a spectral subtype. Its observed $(b-y)=0.60$, implies then $E(b-y) \approx 0.75$ and, assuming that the extinction is standard, an absolute magnitude $M_{V}=-3.5$, in good agreement with the spectral type. This object is almost certainly a Herbig Be star.

\subsubsection{S3R $1 \mathrm{~N} 4$}

A low-resolution spectrum of S3R1N4, taken with the INT, is presented in Fig. 8. $\mathrm{H} \alpha$ is, as in the Asiago spectrum, strongly in emission, with an Equivalent Width $(E W)=-22 \pm 0.5 \AA$. The continuum is only moderately reddened.

A higher resolution spectrum is shown in Fig. 10. From the He I lines, we estimate $v \sin i \approx 320 \mathrm{~km} \mathrm{~s}^{-1}$. The absence of Si III lines suggests a spectral type later than B1. On the other hand, several O II lines are present (in particular $\lambda \lambda$ 4072-4076 ̊), which are not seen after B2. Also, for a later spectral type, Si II $\lambda 4128 \AA$ and $C_{\text {II }} \lambda 4267 \AA$ should be stronger. In the B2V standard, the $\mathrm{O}_{\text {II }}$ lines are weaker than in S3R1N4. Even though $\mathrm{He}_{\mathrm{I}} \lambda 4121 \AA$ is very weak, this is probably related to its Be nature. Therefore we assign a spectral type B1.5Ve to this star. The alternative of making it later but with a higher luminosity would require stronger metallic lines. Therefore $(b-y)=0.28$ implies $E(b-y)=0.39$, putting it at at least the distance to NGC 1893.

Assuming standard absorption, $A_{V}=1.7$ and therefore $M_{V}=-1.7$ if it is a member. This is slightly lower than expected, but not terribly inconsistent with the spectral type (the ZAMS luminosity is $M_{V}=-2.1$ according to Schmidt-Kaler 1982). The photometric parameters of S3R1N4 (Paper I) suggest a later spectral type in the mid-B range. Even though this is typical of early-type Be stars, because the circumstellar disk mimics the colours of a later-type star, the absolute magnitudes of Be stars tend to be higher than those of normal stars of their spectral type.

Given its nearness to S3R1N3, one would be tempted to conclude that S3R1N4 is likely to be a Herbig Be star. However, it must be noted that the spectroscopic and photometric characteristics of S3R1N4 are typical of classical Be stars.

\section{Discussion}

We have selected candidate PMS stars in the area of NGC 1893 covered by the photometry in Paper I because of the presence of emission lines or high reddening. Table 3 lists all the candidates selected, which should be confirmed as PMS stars by studying their spectral energy distribution. All candidates except S4R2N28 have spectral types earlier than G0, which we interpret as a natural consequence of our relatively bright magnitude limit. It is to be expected that a larger population of PMS stars (T Tauri stars) will be detectable at fainter magnitudes. Infrared photometry is an obvious tool to search for this population.

The criterion applied for separation of field stars from PMS members seems to be adequate, since there are very few doubtful cases, namely S4R2N12, which is most likely a field star, and S3R2N6 and S3R2N7, which could be absorption-line PMS members.

The spatial distributions of MS members and PMS candidates can be compared in Fig. 11. Most PMS candidates are concentrated around what could be considered the core of the cluster - the area surrounding the O-type stars S3R1N5 (O7V) and S3R1N16 (O7.5V) just off the rim of the molecular cloud associated with IC 410. There are three PMS stars lying in front 


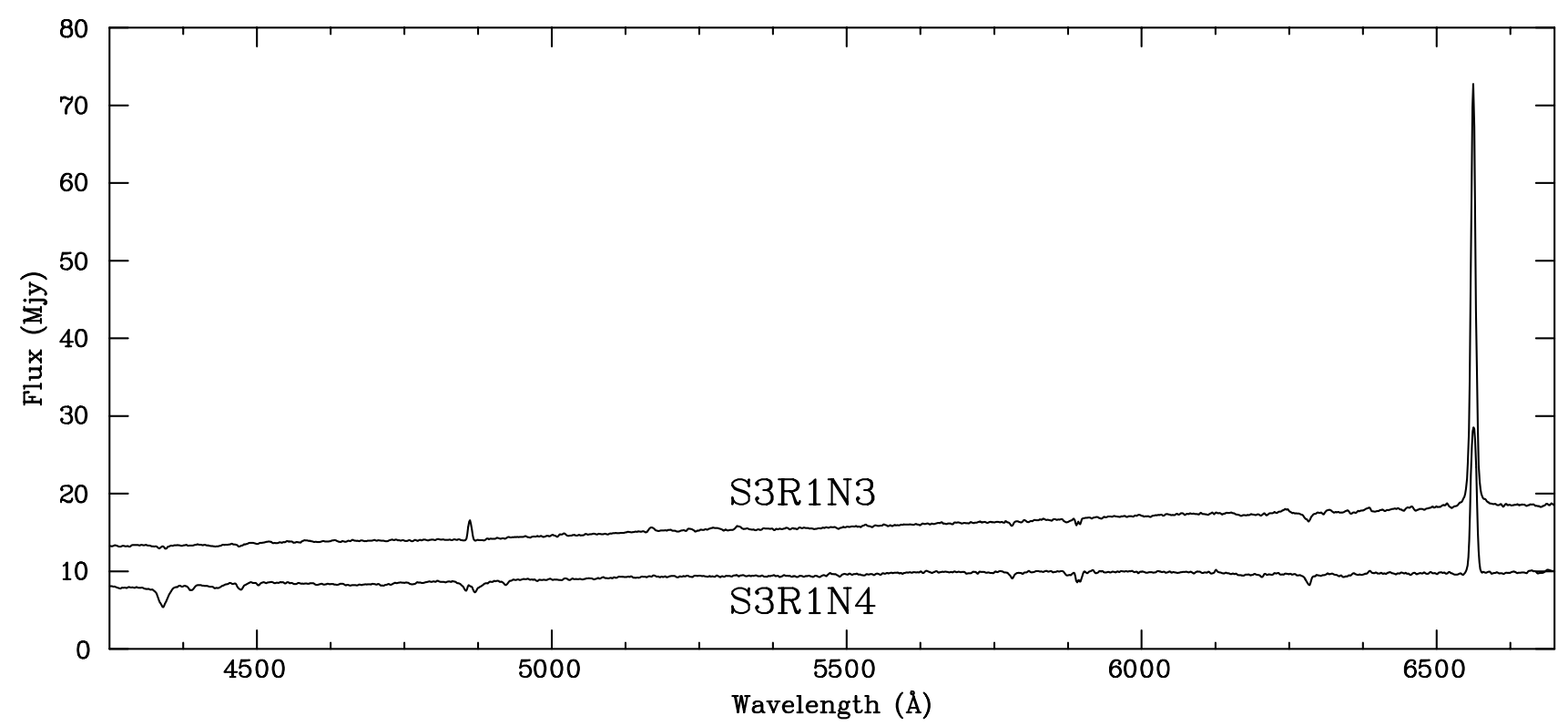

Fig. 8. Low-resolution spectra of S3R1N3 and S3R1N4. A constant value of 10 Mjy has ben added to the spectrum of S3R1N3 for visualization. The flux calibration is not absolute, but the rather higher reddening to S3R1N3 is easily appreciable.

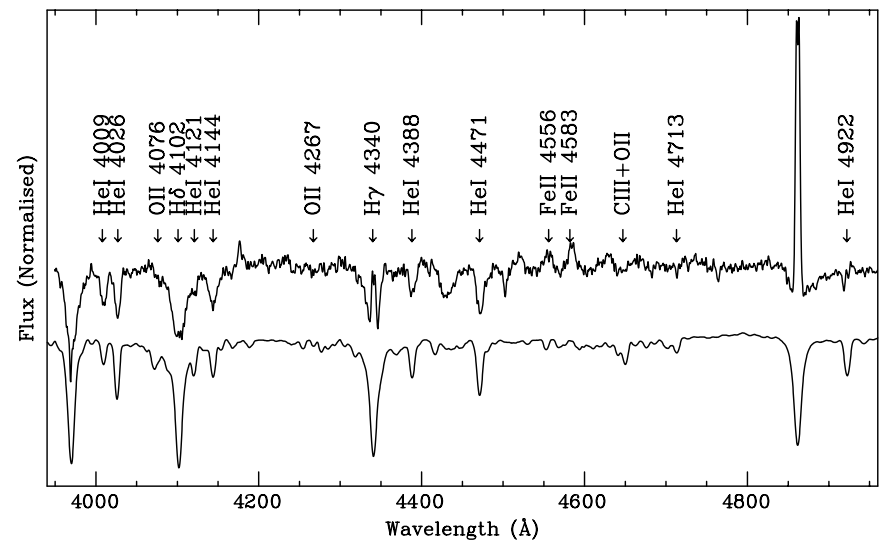

Fig. 9. Blue spectrum of S3R1N3 (top). Interstellar diffuse bands at $\lambda \lambda 4430,4501,4726 \& 4767 \AA$ are not marked. Broad emission feaures are likely to be blends of Fe II lines. The comparison spectrum is that of the B0.5V standard 40 Per, artificially spun up to a rotational velocity of $350 \mathrm{~km} \mathrm{~s}^{-1}$.

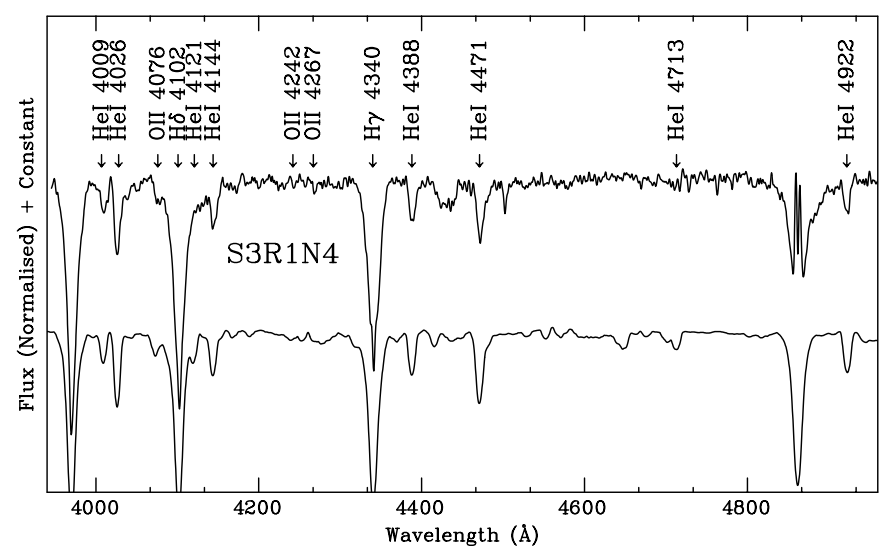

Fig. 10. Blue spectrum of S3R1N4 (top), compared to the spectrum of the B1.5V standard HD 36959 artificially spun up to a rotational velocity $v \sin i=300 \mathrm{~km} \mathrm{~s}^{-1}$.
Table 3. Objects selected as candidate PMS stars. The fourth column indicates whether emission line are present.

\begin{tabular}{lccc}
\hline \hline Star & Spectral type & $E(b-y)$ & Emission? \\
\hline S1R2N4 & A2IV & 0.38 & no \\
S1R2N23 & F6III? & $\sim 0.35$ & yes \\
S2R1N16 & F6V & 0.50 & yes \\
S2R1N26 & F9V & 0.46 & yes \\
S3R1N3 & B0.5IVe & 0.75 & yes \\
S3R1N4 & B1.5Ve & 0.39 & yes \\
S3R1N13 & B5IV & 0.50 & no \\
S3R2N6 & F2V & 0.36 & no \\
S3R2N7 & A9V & 0.24 & no \\
S3R2N12 & A9V & - & no? \\
S4R2N28 & K0V & 0.49 & no \\
\hline
\end{tabular}

of the vast molecular cloud to the West of the cluster (including the Herbig Be star S3R1N3), where there are no MS members. Such situation suggests that this region is in the process of emerging from the parental cloud.

There is only one PMS candidate in the region to the South where a large number of MS members concentrate around the O5V star S3R2N15, suggesting that star formation is already finished in this area. Particularly striking is the dearth of MS members and complete absence of PMS candidates in the vicinity of the O5V star S4R2N17 to the North of the molecular cloud. The surroundings of the fifth O-type star, S3R3N1 (HD 242926), are not covered by our photometry, and therefore have not been included in this work.

Three other PMS candidates, including the emission-line object S1R2N23, are located in the immediate vicinity of the emission nebula Sim 130, to the North of Fig. 11. Some MS members are also found in that region. Only the area to the West of Sim 130 was included in our photometric work, but there are good reasons to believe that the area to the SE of 


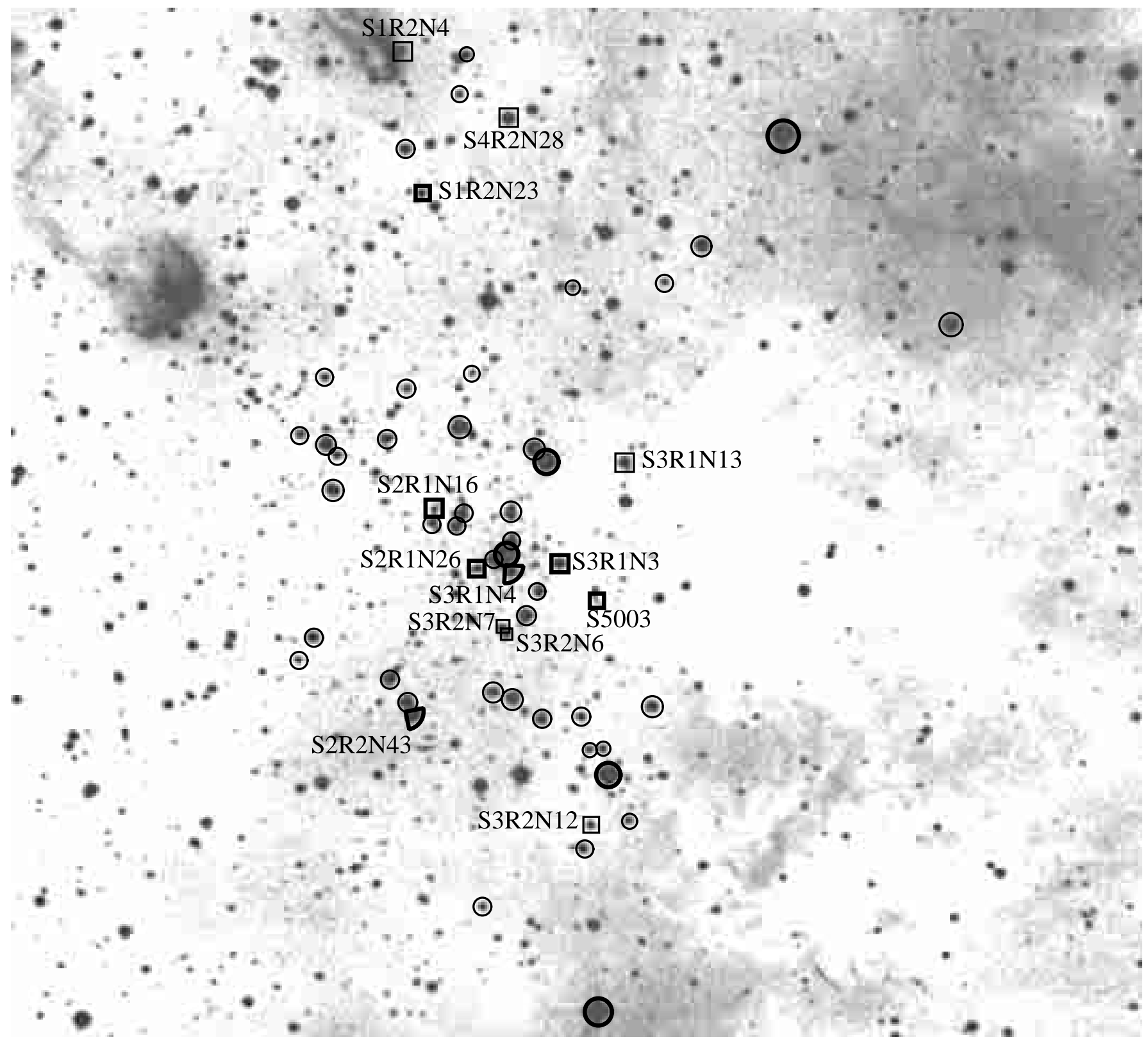

Fig. 11. Chart of the area included in our photometric survey. The image shows a DSS2 red plate of the area. Photometrically confirmed B-type members are marked by thin circles, while O-type members are surrounded by thick circles. Emission-line PMS stars are marked by thick squares, while other candidate PMS stars are indicated by thin squares. The two objects marked with thick triangles are probably classical Be stars. North is up and East is left.

Sim 130 and the surroundings of the pennant nebula Sim 129 (to the NE of the figure) are rich in PMS stars: at least three other emission-line stars are reported in the literature in that area and an IRAS source suggested to be a Young Stellar Object (Campbell et al. 1989) also lies within Sim 129. Because of this, an overall study of massive star formation in NGC 1893 cannot be attempted before this region, which was not covered by our photometry, is explored. This will be the subject of a forthcoming paper.

It is clear, though, that early-type PMS stars in NGC 1893 are only found in two regions: the rim of the molecular cloud and the vicinity of the pennant nebulae Sim 129 and Sim 130. They seem to be absent from regions rich in MS members where there is little gas left. This is consistent with the short
PMS lifetimes for this kind of objects and indicates that massive star formation has already stopped in some areas of the cluster, suggesting that not all stars have formed at the same time.

In this respect, it is interesting to consider the nature of the emission-line B-type objects detected. Even though the characteristics of S3R1N3 clearly support the idea that it is a PMS Herbig Be star, S3R1N4 would certainly be classified as a classical Be star if it was a field object. Moreover, after we reported in Paper I that the B0.5V star S2R2N43 did not have any emission lines, Dr. J. C. Mermilliod has kindly informed us (private communication) that the Be star reported by Schild \& Romanishin (1976) to the South of the cluster centre was without any doubt S2R2N43. Therefore S2R2N43 has lost its 
emission-line characteristics during the last twenty years. This behaviour is typical of classical Be stars, but unprecedented in a Herbig Be star. Although infrared photometry will be necessary to decide on this matter (since classical and Herbig Be stars are well separated in the $(H-K) /(K-L)$ plot; Thé et al. 1994), all this suggests that there are classical Be stars in NGC 1893, while Herbig Be stars are present in the zones where star formation is still active.

\section{Conclusions}

We have identified 5 emission-line stars in the field of NGC 1893 covered by the photometry of Marco et al. (2001), four of which are almost certainly PMS objects. In addition, we find 3 very likely and 3 possible absorption-line PMS stars, selected because they are reddened by an amount higher than or comparable to (respectively) the average for cluster members. One of the PMS stars, S3R1N3, has a spectral type B0.5IVe and it is therefore very young and rather massive. This object, like most other PMS stars is located close to the rim of the molecular cloud associated with the cluster, in the vicinity of the majority of the MS members. The overall picture suggests that the core of NGC 1893 is in the process of emerging from the parental cloud. The rest of the PMS objects lie close to the bright pennant nebulae Sim 129 and Sim 130. No PMS stars are found in the areas of the cluster devoid of gas clouds, suggesting that the population of MS stars in these areas is relatively older.

Acknowledgements. Based in part on observations made at Observatoire de Haute Provence (CNRS), France. We are very grateful to all the staff at the Asiago Observatory for their help during the run. The INT is operated on the island of La Palma by the Isaac Newton Group in the Spanish Observatorio del Roque de Los Muchachos of the Instituto de Astrofísica de Canarias. This research has made use of the Simbad data base, operated at CDS, Strasbourg
(France) and of the WEBDA open cluster database, maintained by Dr. J. C. Mermilliod at the Institute of Astronomy of the University of Lausanne (Switzerland).

The Digitized Sky Surveys were produced at the Space Telescope Science Institute under U.S. Government grant NAG W-2166.

We thank an anonymous referee for helpful comments.

\section{References}

Campbell, B., Persson, S. E., \& Matthews, K. 1989, AJ, 98, 643

Cuffey, J. 1973, AJ, 78, 747

Draper, P. W., Taylor, M., \& Allan, A. 2000, Starlink User Note 139.12, R.A.L.

Gaze, V. F., \& Shajn, G. A. 1952, Izv. Krym. Astrofiz. Obs., 9, 52

Hoag, A. A., Johnson, H. L., Iriarte, B., et al. 1961, Publ. Us. Nav. Obs., XVII, part VII, 347

Howarth, I., Murray, J., Mills, D., \& Berry, D. S. 1998, Starlink User Note 50.21, R.A.L.

Marco, A., Bernabeu, G., \& Negueruela, I. 2001, AJ, 121, 2075 (Paper I)

Massey, P., Johnson, K. E., \& DeGioia-Eastwood, K. 1995, ApJ, 454, 151

Moffat, A. F. J., \& Vogt, N. 1974, Veroeff. Astron. Inst. Bochum, No. 2

Morgan, W. W., Keenan, P. C., \& Kellman, E. 1943, Astrophys. monographs (Univ. Chicago Press)

Popper, D. M. 1980, ARA\&A, 18, 115

Schild, R. E., \& Romanishin, W. 1976, ApJ, 204, 493

Schmidt-Kaler, Th. 1982, in Landolt Börnstein New Ser., vol. 2b, ed. K. Schaifers, \& H. H. Voigt (Springer-Verlag, Berlin)

Shortridge, K., Meyerdicks, H., Currie, M., et al. 1997, Starlink User Note 86.15 , R.A.L

Steele, I. A., Negueruela, I., \& Clark, J. S. 1999, A\&AS, 137, 147

Tapia, M., Costero, R., Echevarría, J., \& Roth, M. 1991, MNRAS, 253, 649

Thé, P. S., de Winter, D., \& Pérez, M. R. 1994, A\&AS, 104, 315

Vacca, W. D., Garmany, C. D., \& Shull, J. M. 1996, ApJ, 460, 914

Vallenari, A., Richichi, A., Carraro, G., \& Girardi, L. 1999, A\&A, 349,825 